\title{
The Resource-Optimized Differentially Modulated Hybrid AF/DF Cooperative Cellular Uplink
}

\author{
Li Wang, Student Member, IEEE and Lajos Hanzo, Fellow, IEEE \\ School of ECS, University of Southampton, SO17 1BJ, UK. \\ Tel: +44-23-8059 3125, Fax: +44-23-8059 4508 \\ Email: \{lw05r, lh\}@ecs.soton.ac.uk; http://www-mobile.ecs.soton.ac.uk
}

\begin{abstract}
In multi-user cellular uplinks cooperating mobiles may share their antennas in order to achieve transmit diversity by formig a virtual antenna array (VAA) in a distributed fashion. In this paper, based on the minimum BER criterion, we investigate cooperating-userselection (CUS) and adaptive-power-allocation (APA) for two types of differentially modulated cooperative cellular uplinks requiring no channel state information (CSI) at the receiver, namely, for the differentialamplify-and-forward (DAF) and the selective differential-decode-andforward (DDF) assisted systems. More specifically, we investigate the cooperative-protocol-selection (CPS) of the uplink system in conjunction with a beneficial CUS as well as the APA scheme in order to further improve the achievable end-to-end performance, leading to a resourceoptimized hybrid cooperative system. Hence, a number of cooperating MSs may be adaptively selected from the available MS candidate pool and the cooperative protocol employed by a specific cooperating MS may also be adaptively selected in the interest of achieving the best possible BER performance.
\end{abstract}

\section{INTRODUCTION}

Multiple antenna aided diversity techniques [1,2] substantially mitigate the deleterious effects of fading, hence improving the end-toend system performance, which is usually achieved by multiple colocated antenna elements at the transmitter and/or receiver. However, it is often impractical for the mobile to employ a large number of antennas for the sake of achieving a diversity gain due to its limited size. Furthermore, owing to the limited separation of the antenna elements, they rarely experience independent fading, which limits the achievable diversity gain and may be further compromised by the detrimental effects of the shadow fading, imposing further signal correlation amongst the antennas in each other's vicinity. Fortunately, in multi-user wireless systems cooperating mobiles may share their antennas in order to achieve uplink transmit diversity by formig a virtual antenna array (VAA) in a distributed fashion. Thus, cooperative diversity relying on the cooperation of the single antenna of multiple terminals may be achieved [3].

On the other hand, in order to carry out classic coherent detection, channel estimation is required at the receiver, which relies on using pilot signals and exploits the fact that in general, the consecutive channel impulse response (CIR) taps are correlated in time as governed by the vehicular speed, i.e. the Doppler frequency. However, channel estimation for an $M$-transmitter, $N$-receiver MIMO system requires the estimation of $(M \times N)$ CIRs, which may impose both an excessive complexity and a high pilot overhead, especially in mobile environments associated with relatively rapidly fluctuating channel conditions. Therefore, in such situations, differentially encoded transmissions combined with non-coherent detection requiring no channel state information (CSI) at the receiver becomes an attractive design alternative, leading to differential modulation assisted cooperative communications [4].

Acknowledgements: The work reported in this paper has formed part of the Core 4 Research Programme of the Virtual Centre of Excellence in Mobile and Personal Communications, Mobile VCE, www.mobilevce.com, whose funding support, including that of EPSRC, is gratefully acknowledged.
It is widely recognized that although a full spatial diversity can be usually achieved by the differentially modulated cooperative system either employing the differential-amplify-and-forward (DAF) or the selective differential-decode-and-forward (DDF) protocol, the achievable end-to-end BER performance may significantly depend on the specific choice of the cooperative protocol employed and/or on the quality of the relay channel. Therefore, in the scenario of the differentially modulated cooperative uplink, where multiple cooperating mobile stations (MSs) are roaming in the area between a specific MS and the base station (BS), an appropriate cooperativeprotocol-selection (CPS) as well as a matching cooperative resource allocation procedure becomes necessary for transmit power allocation and for relay selection in order to maintain a desirable end-to-end performance.

\section{SYSTEM MOdEL}

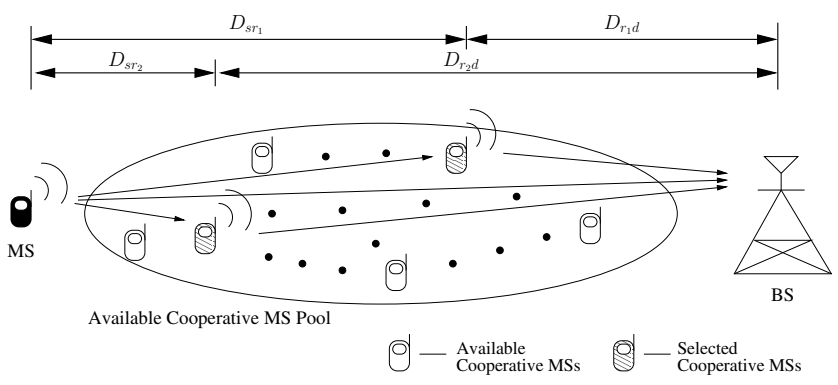

Fig. 1. Cooperation-aided cellular uplink using cooperating-user-selection.

As depicted in Fig. 1, we consider a $U$-user cooperation-aided cellular uplink system, where signal transmission involves two transmission phases, namely, the broadcast phase-I and the relay phase-II. In both phases, any of the well-established multiple access schemes can be employed by the users to guarantee orthogonal transmission among them, such as for example time division multiple access (TDMA), frequency division multiple access (FDMA), or code division multiple access (CDMA). In this paper, TDMA is considered for the sake of simplicity. Furthermore, due to the symmetry of channel allocation among users, we focus our attention on the information transmission of the source MS seen in Fig. 1, which potentially employs $\mathcal{P}_{\text {cand }}=(U-1)$ cooperating MSs in order to achieve cooperative diversity by forming a VAA. Without loss of generality, we simply assume the employment of a single antenna for each terminal, and a unity total power $P$ shared by the collaborating mobiles for transmitting a symbol. Thus, by assuming that $M_{r}$ cooperating MSs are activated out of a total of $\mathcal{P}_{\text {cand }}$, we can express the associated power contraint as: $P=P_{s}+\sum_{m=1}^{M_{r}} P_{r_{m}}$, where $P_{s}$ and $P_{r_{m}}\left(m=1,2, \cdots, M_{r}\right)$ are the transmit power employed by the source MS and the $m$ th RS, respectively. For the sake of simple analytical tractability, we assume that the sum of the 
distances $D_{s r_{u}}$ between the source MS and the $u$ th relay station (RS), as well as that between the $u$ th $\mathrm{RS}$ and the BS, which is represented by $D_{r_{u} d}$, is equal to the distance $D_{s d}$ between the source MS and the BS. Equivalently, as indicated by Fig. 1, we have: $D_{s r_{u}}+D_{r_{u} d}=D_{s d}, u=1,2, \cdots, U-1$. Furthermore, by considering a path-loss exponent of $v$ [5] (we use $v=3$ throughout the paper to simulate a typical urban area), the average channel power gain $\sigma_{i, j}^{2}$ at the output of the channel can be computed according to the inter-node distance $D_{i, j}$ as $\sigma_{i, j}^{2}=D_{i, j}^{-v}, i, j \in\left\{s, r_{u}, d\right\}$, under the assumption that the channel's power gain at the input of the channel is normalized to unity without loss of generality.

In order to avoid channel estimation, the source MS differentially encodes its information symbols $v_{s d}[n] \in \mathcal{M}_{c}=\left\{e^{j 2 \pi m / M} ; m=\right.$ $0,1, \cdots, M-1\}$, each of which contains $\log _{2} M$-bit information, as $s_{s d}[n]=s_{s d}[n-1] v_{s d}[n]$. For the sake of mitigating the impairments imposed by the time-selective channels on the differential transmission, packet-based rather than symbol-based usercooperation is carried out, which is achieved at the expense of both a higher detection delay and increased memory requirements. Hence, the source MS broadcasts a packet constituted of $L_{p}$ differentially encoded DPSK symbols $s_{s d}[n], \quad\left(n=0,1, \cdots, L_{p}-1\right)$ during phase-I, while the BS as well as the RSs receive and store it. In the ensuing phase-II, the DAF or the selective DDF scheme is employed by the RSs, which is the differentially modulated version of the protocol proposed in [3].

Specifically, for the DAF-aided system, the signal received at the $m$ th RS, and that arriving from the $m$ th RS at the BS, can be represented as:

$$
\begin{aligned}
y_{s r_{m}}[n] & =\sqrt{P_{s}} s_{s d}[n] h_{s r_{m}}[n]+w_{s r_{m}}[n] \\
y_{r_{m} d}\left[n+L_{p}\right] & =f_{A M_{r_{m}}} y_{s r_{m}}[n] h_{r_{m} d}\left[n+L_{p}\right]+w_{r_{m} d}\left[n+L_{p}\right],
\end{aligned}
$$

respectively, where the amplification factor $f_{A M_{r_{m}}}$ employed by the $m$ th RS can be specified as in [4], i.e. $f_{A M_{r_{m}}}=\sqrt{\frac{P_{r_{m}}}{P_{s} \sigma_{s r_{m}}^{2}+N_{0}}}$, where $N_{0}$ is the variance of the AWGN imposed at all cooperating MSs as well as at the BS. As to the selective DDF-aided system, where the activated RS will make sure that the information contained in the packet received from the source MS can be correctly recovered by differentially decoding the received signal with the aid of CRC checking prior to forwarding it to the $\mathrm{BS}$, the signal received from the $m$ th RS at the BS can be expressed as:

$$
y_{r_{m} d}\left[n+L_{p}\right]= \begin{cases}\sqrt{P_{r_{m}}} s_{s d}[n] h_{r_{m} d}\left[n+L_{p}\right], & \text { if } \Phi_{1}^{m} \\ 0, & \text { if } \Phi_{2}^{m},\end{cases}
$$

where $\Phi_{1}^{m}$ is defined as the first scenario, when the $m$ th activated cooperating MS perfectly recovers the information received from the source MS and thus transmits the differentially remodulated signal to the BS. By contrast, $\Phi_{2}^{m}$ is defined as the second scenario, when the $m$ th activated cooperating MS fails to correctly decode the signal received from the source MS and hence remains silent during the relaying phase. Therefore, the scenarios $\Phi_{1}^{m}$ and $\Phi_{2}^{m}$ can be simply represented as:

$$
\begin{aligned}
& \Phi_{1}^{m} \triangleq\left\{P_{r_{m}} \neq 0\right\}, \\
& \Phi_{2}^{m} \triangleq\left\{P_{r_{m}}=0\right\} .
\end{aligned}
$$

Additionally, in order to further mitigate the performance degradation induced by the mobility of the cooperating MSs, the multiplesymbol differential sphere detection (MSDSD) proposed for noncooperative direct transmission in [6] was specifically redesigned in [7] for the cooperative system. Therefore, the MSDSD is assumed to be employed at the RS and the BS in our simulations, unless otherwise specified.

\section{Performance Analysis of the Cooperative Uplink}

It was demonstrated in $[6,7]$ that the Doppler-frequency-induced error floor encountered by the conventional differential detection (CDD) (or equivalently by the MSDSD using an observation window size of $N_{\text {wind }}=2$ ) is expected to be completely eliminated by jointly detecting $N_{\text {wind }}>2$ consecutive received symbols with the aid of the MSDSD, provided that $N_{\text {wind }}$ is sufficiently high. Therefore, under the assumption that the associated performance degradation can be mitigated by the MSDSD in both the DAF- and selective DDF-aided cooperative system, it is reasonable to expect that the BER performance exhibited by the cooperative system employing the MSDSD in a relatively rapidly fading environment can be closely approximated by that achieved by its CDD-aided counterpart in a slow-fading channel. Hence, in the ensuing subsections our performance analysis of both the DAF- and DDF-aided systems is carried out without considering the detrimental effects imposed by the mobility of the MSs. Consequently, our task may be interpreted as the performance analysis of a CDD-assisted differentially modulated cooperative system operating in slow-fading channels.

\section{A. Classic Unified Approach to the Performance Analysis}

A unified approach to the probability of error for differential modulated direct transmission systems discussed in [8] is also applicable to the theoretical BER performance analysis of both the DAF- and DDF-aided cooperative systems, which is summarized as follows:

Step 1: According to [8], determine the error probability expression conditioned on the received SNR.

Step 2: Obtain the expression of the received signal-to-noise ratio (SNR) per bit, which is a function of the channel gain.

Step 3: Average the above-mentioned conditional error probability over the entire range of received SNR per bit values by weighting it according to its probability of occurrence with the aid of its probability density function (PDF).

\section{B. Theoretical Analysis of the DDF-Aided Cooperative Uplink}

In the selective DDF-aided cooperative uplink, some of the $M_{r}$ cooperating MSs may not actively participate during the relaying phase, for the sake of avoiding the potential error propagation imposed by error-infested the imperfect signal recovery. By simply assuming that the transmission packet length is sufficiently high with respect to the channel's coherent time, the worst-case packet loss ratio (PLR) at the $m$ th cooperating MS can be expressed as:

$$
P_{P L R_{m}, \text { upper }}=1-\left(1-P_{S E R_{m}}\right)^{L_{p}}
$$

for a given packet length $L_{p}$, where $P_{S E R_{m}}$ represents the symbol error rate (SER) at the $m$ th cooperating MS, which can be calculated as [9]:

$$
P_{S E R_{m}}=\frac{M-1}{M}+\frac{\left|\rho_{m}\right| \tan \left(\frac{\pi}{\mathcal{M}_{c}}\right)}{\xi\left(\rho_{m}\right)}\left[\frac{1}{\pi} \arctan \left(\frac{\xi\left(\rho_{m}\right)}{\left|\rho_{m}\right|}\right)-1\right],
$$

where $\rho_{m}$ and the function $\xi(x)$, respectively, can be written as follows:

$$
\begin{aligned}
\rho_{m} & =\frac{P_{s} \sigma_{s r_{m}}^{2}}{N_{0}} /\left(1+\frac{P_{s} \sigma_{s r_{m}}^{2}}{N_{0}}\right), \\
\xi(x) & =\sqrt{1-|x|^{2}+\tan ^{2}(\pi / M)} .
\end{aligned}
$$

Then, based on the $P_{P L R_{m}}$, upper expression of Eq.(6), the average end-to-end BER upper bound of a DDF-aided cooperative system can be obtained. For simplicity, we consider the system where only $M_{r}=$ 1 cooperating user is activated to parcipate in relaying the signal 
from the source MS to the BS as an example. The conditional worstcase end-to-end BER, $P_{B E R, u p p e r \mid}^{D D b}$, is obtained by the weighted summation of the two-component conditional BERs corresponding to the scenarios $\Phi_{1}^{1}$ of Eq. (4) and $\Phi_{2}^{1}$ of Eq. (5) as:

$$
\begin{aligned}
P_{B E R, \text { upper } \mid \gamma^{b}}^{D D F}=\left(1-P_{P L R_{1}, \text { upper }}\right) P_{B E R \mid \gamma_{\Phi_{1}}^{b}}^{\Phi_{1}^{1}} & \\
& +P_{P L R_{1}, \text { upper }} P_{B E R \mid \gamma_{\Phi_{2}}^{b}}^{\Phi_{2}^{1}},
\end{aligned}
$$

where

$$
P_{B E R \mid \gamma_{\Phi_{i}^{1}}^{b}}^{D A F}=\frac{1}{2^{2 L} \pi} \int_{-\pi}^{\pi} f\left(a, b, M_{r}+1, \theta\right) e^{-\alpha(\theta) \gamma_{\Phi_{i}^{1}}^{b}} d \theta
$$

in which

$$
\begin{gathered}
f(a, b, L, \theta)=\frac{b^{2}}{2 \alpha(\theta)} \sum_{l=1}^{L}\left(\begin{array}{c}
2 L-1 \\
L-l
\end{array}\right)\left[\left(\beta^{-l+1}-\beta^{l+1}\right)\right. \\
\left.\times \cos ((l-1)(\theta+\pi / 2))-\left(\beta^{-l+2}-\beta^{l}\right) \cos (l(\theta+\pi / 2))\right], \\
\alpha(\theta)=\frac{b^{2}\left(1+2 \beta \sin \theta+\beta^{2}\right)}{2},
\end{gathered}
$$

and

$$
\beta=a / b \text {. }
$$

In Eq. (11) the parameters $a$ and $b$ are the modulation-dependent factors defined in [10]. For example, we have $a=\sqrt{2-\sqrt{2}}$ and $b=\sqrt{2+\sqrt{2}}$ for DQPSK modulation using Gray coding. Moreover, the parameter $L$ of Eq. (12) denotes the number of diversiy paths. For example, when $M_{r}$ cooperating MSs are activated, we have $L=$ $M_{r}+1$, assuming that the BS combines the signals received from all the $M_{r}$ RSs as well as that from the direct link.

On the other hand, since in our performance analysis the MRC scheme is assumed to be employed at the BS to combine the signals potentially forwarded by multiple cooperating MSs and the signal transmitted from the source MS prior to the CDD, the received SNR per bit after the MRC stage is simply the sum of that of each combined path, which is expressed as:

$$
\begin{aligned}
\gamma_{\Phi_{1}^{1}}^{b} & =\gamma_{s d}^{b}+\gamma_{r_{1} d}^{b}, \\
\gamma_{\Phi_{2}^{1}}^{b} & =\gamma_{s d}^{b} .
\end{aligned}
$$

Therefore, following the method outlined in Section III-A, the unconditional BER of the scenario $\Phi_{1}$ can be computed by averaging the conditional BER over the fading distribution of the SNR per bit at the output of the MRC with the aid of its PDF as follows [8]:

$$
\begin{aligned}
P_{B E R}^{\Phi_{1}^{1}} & =\int_{-\infty}^{\infty} P_{B E R \mid \gamma_{\Phi_{1}^{1}}^{b}} \cdot p_{\gamma_{\Phi_{1}^{1}}^{b}}(\gamma) d \gamma \\
& =\frac{1}{2^{2 L} \pi} \int_{-\pi}^{\pi} f(a, b, L=2, \theta) \int_{-\infty}^{\infty} e^{-\alpha(\theta) \gamma_{\Phi_{1}^{1}}^{b}} p_{\gamma_{\Phi_{1}^{1}}^{b}}(\gamma) d \gamma d \theta \\
& =\frac{1}{2^{2 L} \pi} \int_{-\pi}^{\pi} f(a, b, L=2, \theta) \mathcal{M}_{\gamma_{\Phi_{1}^{1}}^{b}}(\theta) d \theta
\end{aligned}
$$

where the joint MGF of the received SNR per bit recorded at the BS for the scenario $\Phi_{1}^{1}$ is expressed as:

$$
\begin{aligned}
& \mathcal{M}_{\gamma_{\Phi_{1}^{b}}^{b}}(\theta) \\
& =\int_{-\infty}^{\infty} \int_{-\infty}^{\infty} e^{-\alpha(\theta)\left(\gamma_{s d}^{b}+\gamma_{r_{1} d}^{b}\right)} p_{\gamma_{s d}^{b}}\left(\gamma_{s d}\right) p_{\gamma_{r_{1} d}^{b}}\left(\gamma_{r_{1} d}\right) d \gamma_{s d} d \gamma_{r_{1} d} \\
& =\frac{N_{0}^{2}}{\left(N_{0}+\alpha(\theta) P_{s} \sigma_{s d}^{2}\right)\left(N_{0}+\alpha(\theta) P_{r_{1}} \sigma_{r_{1} d}^{2}\right)}
\end{aligned}
$$

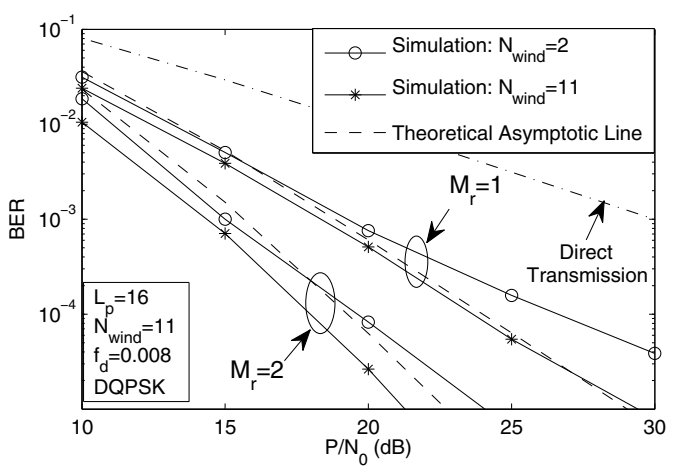

Fig. 2. BER performance of the DQPSK modulated selective DDF-aided cooperative cellular systems having $M_{r}$ activated cooperating MSs in a Rayleigh fading channel associated with $f_{d}=0.008$ and $v=3$.

with $p_{\gamma_{s d}^{b}}\left(\gamma_{s d}\right)$ and $p_{\gamma_{r_{d} b}^{b}}\left(\gamma_{r_{1} d}\right)$, respectively, denoting the PDF of the received SNR per bit for the direct link and for the relay-todestination link. Under the assumption of Rayleigh fading channels, both of these expressions are given as [5]:

$$
p_{\gamma^{b}}(\gamma)=\left\{\begin{array}{ll}
\frac{1}{\overline{\gamma^{b}}} \exp \left(-\frac{\gamma}{\gamma^{b}}\right), & \gamma \geq 0 \\
0, & \gamma<0
\end{array},\right.
$$

where $\overline{\gamma^{b}}$ denotes the average received SNR per bit. In parallel, the unconditional BER of the scenario $\Phi_{2}^{1}$ can be obtained as:

$$
\begin{aligned}
P_{B E R}^{\Phi_{2}^{1}} & =\int_{-\infty}^{\infty} P_{B E R \mid \gamma_{\Phi_{2}^{1}}^{b}} \cdot p_{\gamma_{\Phi_{2}^{1}}^{b}}(\gamma) d \gamma, \\
& =\frac{1}{2^{2 L} \pi} \int_{-\pi}^{\pi} f(a, b, L=1, \theta) \mathcal{M}_{\gamma_{\Phi_{2}^{\frac{1}{2}}}^{b}}(\theta) d \theta,
\end{aligned}
$$

where the MGF of the received SNR per bit recorded at the BS for the scenario $\Phi_{2}$ is written as:

$$
\begin{aligned}
\mathcal{M}_{\gamma_{\Phi \frac{1}{b}}^{b}}(\theta) & =\int_{-\infty}^{\infty} e^{-\alpha(\theta) \gamma_{s d}^{b}} p_{\gamma_{s d}^{b}}\left(\gamma_{s d}\right) d \gamma_{s d}, \\
& =\frac{N_{0}}{N_{0}+\alpha(\theta) P_{s} \sigma_{s d}^{2}} .
\end{aligned}
$$

Finally, in the light of Eq. (10), the unconditional worst-case end-toend BER can be computed as:

$$
P_{B E R, \text { upper }}^{D D F}=\left(1-P_{P L R_{1}, \text { upper }}\right) P_{B E R}^{\Phi_{1}^{1}}+P_{P L R_{1}, \text { upper }} P_{B E R}^{\Phi_{2}^{1}} .
$$

Similarly, the BER upper bound can also be attained for the selective DDF-aided cooperative systems relying on $M_{r}>1$ RSs.

Since the worst-case BER expression derived for the selective DDF-aided system does not take the negative impact of the timeselective channel into account, the resultant asymptotic line may not be capable of accurately approximating the true achievable BER performance of a DDF-aided system employing the CDD in a rapidly fading environment. However, with the aid of the MSDSD [6, 7] using $N_{\text {wind }}>2$, the performance loss induced by the relative mobility of the cooperating terminals and the BS can be significantly reduced. Thus, as revealed by Fig. 2, the worst-case BER bound adequately captures the dependency of the system's BER on the $P / N_{0}$ ratio.

\section{Theoretical Analysis of the DAF-Aided Cooperative Uplink}

Since the theoretical BER expression of the DAF-aided cooperative system employing the MRC and CDD schemes has already been derived in [11] following the method outlined in Section III-A, we will not repeat the detailed derivation here, we simply provide the 
final result. According to [11], a high-SNR-based tight BER lower bound for the DAF-aided cooperative system can be represented as:

$$
\begin{aligned}
P_{B E R, h i g h-s n r}^{D A F}(a, b, & \left.M_{r}\right) \gtrsim \frac{F\left(a, b, M_{r}+1\right) N_{0}^{M_{r}+1}}{P_{s} \sigma_{s d}^{2}} \\
\times & \prod_{m=1}^{M_{r}} \frac{P_{r_{m}} \sigma_{r_{m}, d}^{2}+P_{s} \sigma_{s r_{m}}^{2} Z_{r_{m}, m i n}}{P_{s} P_{r_{m}} \sigma_{s r_{m}}^{2} \sigma_{r_{m} d}^{2}},
\end{aligned}
$$

where we have [11]

$$
F(a, b, L)=\frac{1}{2^{2 L} \pi} \int_{-\pi}^{\pi} \frac{f(a, b, L, \theta)}{\alpha^{L}(\theta)} d \theta,
$$

and $Z_{r_{m}, \text { min }}$ is given by Eq. (22) in [11]. Importantly, the BER lower-bound of (28) implies that a DAF-aided cooperative system having $M_{r}$ activated RSs is capable of achieving a diversity order of $L=\left(M_{r}+1\right)$, as indicated by the exponent $L$ of the noise variance.

\section{Resource-Optimized DifFEREnTIALly Modulated Hybrid CoOperative CEllular UPLINK}

\section{A. Resource Optimization for the Cooperative Uplink}

With the aid of the theoretical BER bound expressions of Eqs. (27) and (28) derived for the DDF- and DAF-assisted cooperative uplinks, respectively, both the transmit power and the RS locations can be optimized by using the minimum BER criterion. Specifically, the optimized location of the cooperating users, expressed in terms of the normalized distance $d_{m}=D_{s r_{m}} / D_{s d},\left(m=1,2, \cdots, M_{r}\right)$ between the source MS and the RS, which is the one minimizing Eqs. (27) or (28), can be found numerically for a given power allocation $c_{m}=P_{r_{m}} / P_{s}$, and vice versa. However, the results may not be the global optimum in terms of the best achievable BER performance. Hence, for the sake of attaining the globally optimum location, an iterative power-versus-RS-location optimization process has to be performed as follows:

Step 1: Initialize the starting point $\left(\left\{c_{m}\right\}_{m=1}^{M_{r}},\left\{d_{m}\right\}_{m=1}^{M_{r}}\right)$ for the search in the $2 M_{r}$-dimensional space, hosting the $M_{r}$ powers and RS locations.

Step 2: Calculate the locally optimum location $\left\{d_{m, l o c a l}\right\}_{m=1}^{M_{r}}$ of the cooperating users for the current power allocation, $\left\{c_{m}\right\}_{m=1}^{M_{r}}$.

Step 3: If we have $\left\{d_{m, \text { local }}\right\}_{m=1}^{M_{r}} \neq\left\{d_{m}\right\}_{m=1}^{M_{r}}$, then let $\left\{d_{m}\right\}_{m=1}^{M_{r}}=\left\{d_{m, l o c a l}\right\}_{m=1}^{M_{r}}$. Otherwise, stop the search, since the globally optimum solution has been found: $\left\{d_{m, \text { globle }}\right\}_{m=1}^{M_{r}}=\left\{d_{m, \text { local }}\right\}_{m=1}^{M_{r}}$ and $\left\{c_{m, \text { globle }}\right\}_{m=1}^{M_{r}}=$ $\left\{c_{m}\right\}_{m=1}^{M_{r}}$.

Step 4: Calculate the locally optimum power allocation $\left\{c_{m, l o c a l}\right\}_{m=1}^{M_{r}}$ of the cooperating RSs for the current location, $\left\{d_{m}\right\}_{m=1}^{M_{r}}$.

Step 5: If we have $\left\{c_{m, l o c a l}\right\}_{m=1}^{M_{r}} \neq\left\{c_{m}\right\}_{m=1}^{M_{r}}$, then let $\left\{c_{m}\right\}_{m=1}^{M_{r}}=\left\{c_{m, l o c a l}\right\}_{m=1}^{M_{r}}$ and return to Step1. Otherwise, stop the search, since the globally optimum solution has been found: $\left\{d_{m, \text { globle }}\right\}_{m=1}^{M_{r}}=\left\{d_{m, \text { local }}\right\}_{m=1}^{M_{r}}$ and $\left\{c_{m, \text { globle }}\right\}_{m=1}^{M_{r}}=\left\{c_{m}\right\}_{m=1}^{M_{r}}$.

Since it is likely that no available cooperating MS candidate is situated in the exact optimum location found by the above-mentioned optimization, our proposed cooperating-user-selection (CUS) scheme simply chooses the available MS that roams closest to the optimum location and then carries out the adaptive power allocation (APA). The rationale of the CUS scheme is based on the observation that the achievable BER is proportional to the distance between the cooperating MS and the optimum location, as indicated in Figs. 4(a) and 5(a), where the BER is portrayed versus $d_{m}$ for both the DAF- and selective DDF-aided cooperative uplinks activating $M_{r}$

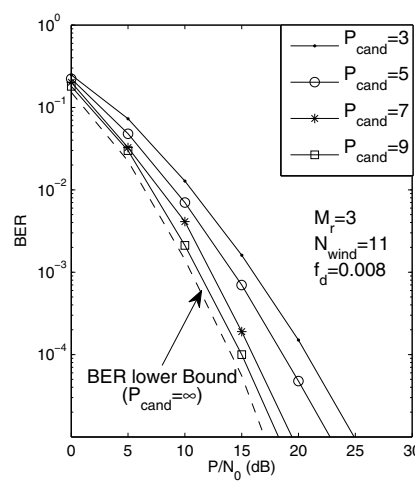

(a) BER performance

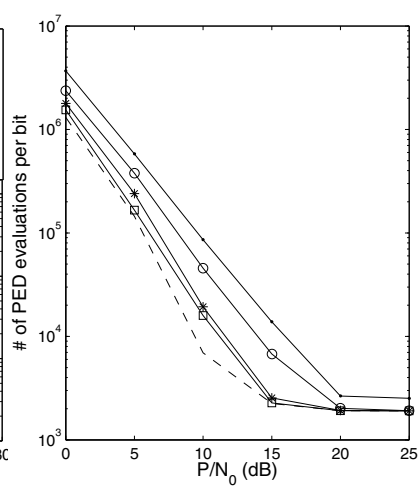

(b) Complexity imposed by the MSDSD
Fig. 3. The effects of the size of the cooperating RS pool on the DAF-aided DQPSK modulated user-cooperative cellular uplink employing the CUS and APA schemes in a Rayleigh fading channel associated with $f_{d}=0.008$ and $v=3$, where $M_{r}=3$ cooperating users are activated.

cooperating RSs. Furthermore, it is worth emphasizing that the above optimization process requires an "off-line" operation. Hence, its complexity does not contribute to the complexity of the real-time CUS scheme.

In order to investigate the impact of the candidate pool size $\mathcal{P}_{\text {cand }}$ on the end-to-end BER performance of the cooperative system employing our proposed CUS and the APA schemes, the BER curves corresponding to different values of $\mathcal{P}_{\text {cand }}$ are plotted versus the transmit SNR, $P / N_{0}$ in Fig. 3(a) for the DAF-aided uplink as an example, where $M_{r}=3$ out of $\mathcal{P}_{\text {cand }}$ cooperating MSs are activated. Without loss of generality, we simply assume that the locations of all the cooperating candidates are independent and uniformly distributed along the direct LOS link connecting the source MS and the BS, which are expected to change from time to time. Interestingly, despite having a fixed number of activated cooperating MSs, the end-to-end BER performance of the DAF-aided system steadily improves and approaches that of the idealized benchmark system upon increasing the value of $\mathcal{P}_{\text {cand }}$, as observed in Fig. 3(a). On the other hand, it can be seen in Figure 3(b) that the higher the number of cooperating candidates, the lower the computational complexity imposed by the MSDSD at the BS. Specifically, observe in Fig. 3 that by increasing the size of the candidate pool from $\mathcal{P}_{\text {cand }}=3$ to 9 , a performance gain of about $7 \mathrm{~dB}$ can be attained, while simultaneously achieving a detection complexity reduction factor of 6.5 at the target BER of $10^{-5}$. In comparison to the idealized scenario, where an inifinite number of cooperating candidates are assumed to be independently and uniformly distributed between the source MS and the BS, the DAF-aided cooperative system using both the CUS and APA schemes only suffers a negligible performance loss, when having $\mathcal{P}_{\text {cand }}=9$ cooperating candidates. Therefore, the benefits brought about by the employment of the CUS and APA schemes may be deemed substantial in a typical cellular uplink.

\section{B. Comparison of the DAF- and DDF-Aided Cooperative Uplinks}

The BER performance of both the DAF- and DDF-aided cooperative system employing the APA scheme is depicted versus $d_{m}$ in Figs. 4(a) and 5(a), respectively, in comparison to that of the system employing the simple equal power allocation. We simply assume that multiple activated cooperating users are located at the same distance from the source user. Observe in Figs. 4(a) and 5(a) that significant performance improvements can be achieved by the APA scheme for both the DAF- and DDF-aided systems, when the cooperating user 


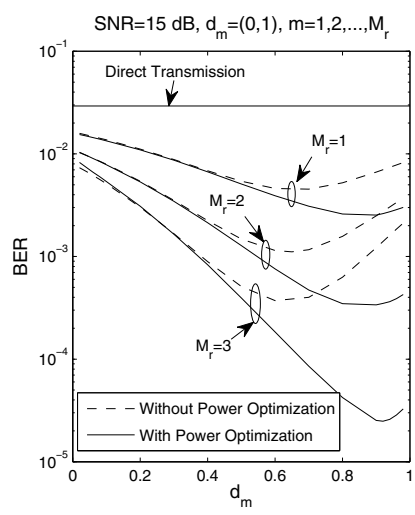

(a) Adaptive power allocation

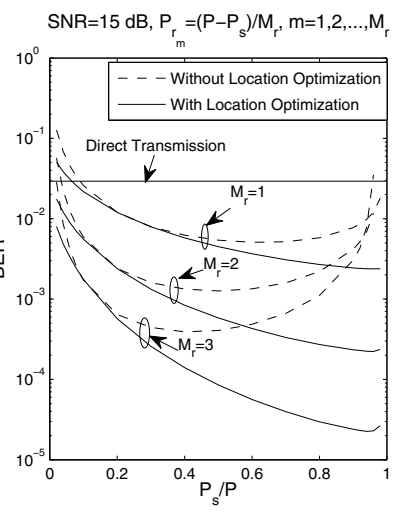

(b) Relay location optimization
Fig. 4. Power and relay location optimization for DQPSK modulated DAFaided cooperative cellular systems having $M_{r}$ activated RSs in a Rayleigh fading channel associated with $v=3$. The BER was computed from Eq. (28).

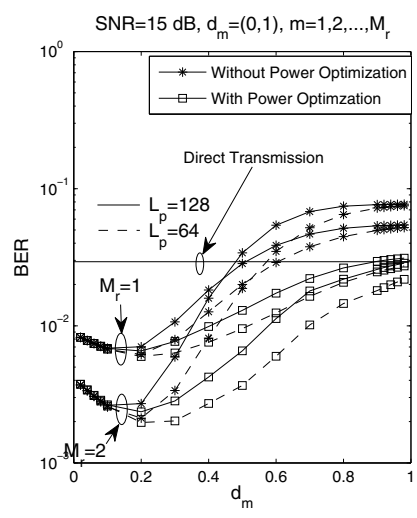

(a) Adaptive power allocation

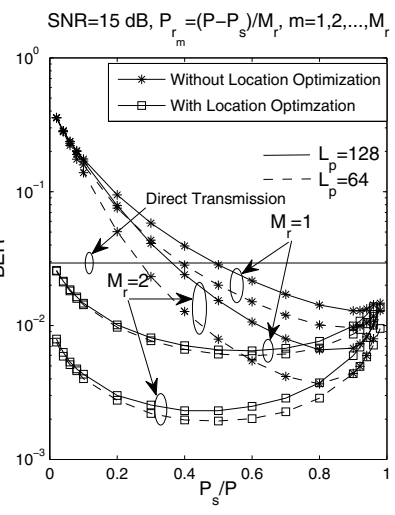

(b) Relay location optimization
Fig. 5. Power and RS location optimization for the DQPSK modulated DDFaided cooperative cellular systems having $M_{r}$ activated RSs in a Rayleigh fading channel associated with $v=3$. The BER was computed from Eq. (27).

is situated closer to the BS than to the source MS. However, due to the difference between the relaying mechanisms employed by the two above-mentioned cooperative systems, it is interesting to observe that the BER trends seen in Fig. 4(a) are quite different from those emerging from Fig. 5(a). Specifically, Fig. 4(a) demonstrates that it is desirable to choose multiple cooperating users closer to the BS than to the source MS in a DAF-aided cooperative system, espcially when optimally sharing the power among the cooperating users. By contrast, Fig. 5(a) reveals that the cooperating MSs roaming in the vicinity of the source MS are preferred for a DDF-aided system in the interest of maintaining a better BER performance. Additionally, by comparing the results of Figs. 4(a) and 5(a), we found that the DDF-aided system suffers from a less dramatic performance loss than its DAF-aided counterpart by employing the equal-power allocation scheme, if the multiple cooperating MSs are closer to their desired locations. Hence, for the sake of reducing the complexity, the DDF-aided cooperative system may simply employ an equalpower allocation, while being still capable of achieving a desirable performance with the aid of the CUS scheme.

In parallel, the BER performance of the DAF- and DDF-aided systems is depicted against $P_{s} / P$ in Figs. 4(b) and 5(b), respectively. A significant performance gain can be attained by locating the cooperating MS at the optimum position, rather than in the middle of the source MS and BS path for both systems, which is expected to become even higher, as the number of actively cooperating MSs,

\begin{tabular}{|c|c|c|}
\hline & DAF-Aided Uplink & DDF-Aided Uplink \\
\hline Overall Performance & $\begin{array}{r}\text { Better when SR } \\
\text { link quality is poor }\end{array}$ & $\begin{array}{r}\text { Better when SR } \\
\text { link guality is good }\end{array}$ \\
\hline Overall Complexity & $\begin{array}{r}\text { Relatively low, no } \\
\text { decoding at RSs }\end{array}$ & $\begin{array}{l}\text { Relatively high, decoding } \\
\text { and re-encoding at RSs }\end{array}$ \\
\hline $\begin{array}{l}\text { Performance's Sensitivity to } \\
\text { Source-Relay Link Quality }\end{array}$ & Relatively moderate & Strong \\
\hline $\begin{array}{l}\text { Performance's Sensitivity } \\
\text { to Packet Length } L_{p}\end{array}$ & Insensitive & $\begin{array}{l}\text { Strong without CUS, } \\
\text { minor with CUS }\end{array}$ \\
\hline Desirable RS Locations & Near the BS & Near the source MS \\
\hline $\begin{array}{l}\text { Desirable Transmit Power } \\
\text { for the Source MS }\end{array}$ & $\begin{array}{l}\text { About } 88 \% \text { of } \\
\text { the total power }\end{array}$ & $\begin{array}{l}\text { About } 60 \% \text { of } \\
\text { the total power }\end{array}$ \\
\hline $\begin{array}{l}\text { Worst Case Performance } \\
\text { (Bad Resource Allocation) }\end{array}$ & $\begin{array}{l}\text { Slightly better than the } \\
\text { non-cooperative system }\end{array}$ & $\begin{array}{l}\text { Significantly worse than } \\
\text { the non-cooperative system }\end{array}$ \\
\hline Importance of CUS and APA & Equally important & $\begin{array}{r}\text { CUS is significantly } \\
\text { more important }\end{array}$ \\
\hline
\end{tabular}

TABLE I

COMPARISON BETWEEN THE DAF- AND DDF-AIDED COOPERATIVE CELLULAR UPLINKS

$M_{r}$, increases. For optimum cooperating user location, instead of allocating the majority of the total transmit power to the source MS - as it was suggested by Fig. 4(b) for the DAF-aided system in the interest of achieving an improved BER performance - the results of Fig. 5(b) suggest that only about half of the total power has to be assigned to the source MS, if the DDF scheme is used. Furthermore, the information bit stream is CCITT- 4 coded by the source MS for the DDF-aided system in order to carry out the CRC checking at the cooperating MS with the aid of a 4-bit CRC sequence. Hence, for the sake of maintaining a relatively high effective throughput, two different transmission packet lengths are used, namely, $L_{p}=128$ and $L_{p}=64$ DQPSK symbols. However, a larger value of $L_{p}$ may result in a worse end-to-end performance, since the activated RS is less likely to participate in signal forwarding owing to the increased packet loss ratio. Fortunately, as observed in Figs. 5(a) and 5(b), the originally significant performance differences caused by the different packet lengths of $L_{p}=128$ and $L_{p}=64$, can be substantially reduced for the DDF-aided system, provided that the cooperating user is situated at the optimum location. Finally, the comparison of the DAF- and DDF-aided systems is summarized in Table I.

\section{Resource-Optimized Hybrid Cooperative Cellular Uplink}
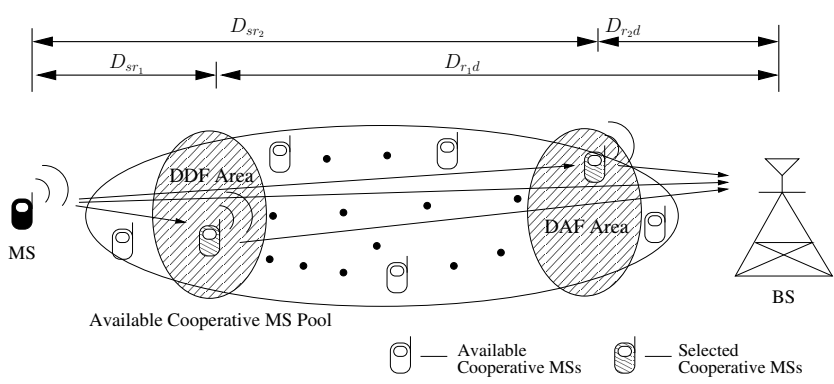

Fig. 6. Cooperative uplink systems using the joint CPS and CUS scheme.

From our comparison of the DAF- and DDF-aided cooperative systems in Section IV-B, we realized that the two above-mentioned relaying mechanisms have complementary characteristics, reflected for example, by their distinct optimum cooperative resource allocations. In the light of the complementarity of the two relaying schemes, a more flexible cooperative scenario can be created, where either the DAF or DDF schemes are activated in the interest of enhancing the achievable performance of the cooperative system, while maintaining a moderate complexity. In contrast to the conventional cooperative 
system employing a single cooperative mechanism, the cooperating MSs roaming in different areas between the source MS and the BS may be activated and the relaying schemes employed by each activated RS may be adaptively selected, for the sake of achieving the best possible performance. For the sake of simplicity, let us now consider the hybrid cooperative cellular uplink employing the joint CPS and CUS scheme, as portrayed in Fig. 6, where $M_{r}=2$ cooperating MSs roaming in the preferred DDF- and DAF-RS-area are activated, in order to forward the source MS's information to the BS. The particular cooperative protocol employed by the activated RSs is determined according to the specific area which they happen to be situated in. In order to make the most of the complementarity of the DAF and DDF schemes, it may be assumed that one of the cooperating MSs is activated in the preferred area of the DAF-RS, while the other from the 'DDF-area', although naturally, there may be more than one cooperating MSs roaming within a specific desirable area. Then, under the assumption that the first selected cooperating MS is roaming in the 'DDF-area', while the second one is roaming in the 'DAF-area', the cooperative resource allocation is optimized as shown in Table II based on the minimum BER criterion. Due to lack of space, the derivation of the theoretical BER for the hybrid cooperative system is omitted, which is similar to that for the DAFor DDF-aided system in Section III. As expected, Table II reveals that the 'DDF-area' and the 'DAF-area' are still located in the vicinity of the source MS and the BS, respectively. Additionally, the majority of the total transmit power, i.e. about $70 \%$, should be allocated to the source MS, while $\frac{2}{3}$ of the remaining power should be assigned to the cooperating MS roaming in the 'DDF-area'.

\begin{tabular}{|c|c|c|c|}
\hline$M_{r}$ & $P / N_{0}(\mathrm{~dB})$ & {$\left[P_{s}, P_{r_{1}}, \cdots P_{r_{M_{r}}}\right]$} & {$\left[d_{1}, d_{2} \cdots d_{M_{r}}\right]$} \\
\hline \multirow{3}{*}{2} & 10 & {$[0.702,0.202,0.096]$} & {$[0.26,0.86]$} \\
\hline & 20 & $0.702,0.202,0.096$ & $0.31,0.86$ \\
\hline & 30 & {$[0.702,0.202,0.096]$} & {$[0.31,0.91]$} \\
\hline
\end{tabular}

TABLE II

Resource Allocation FOR THE HyBRID COOPERATIVE UPLINK

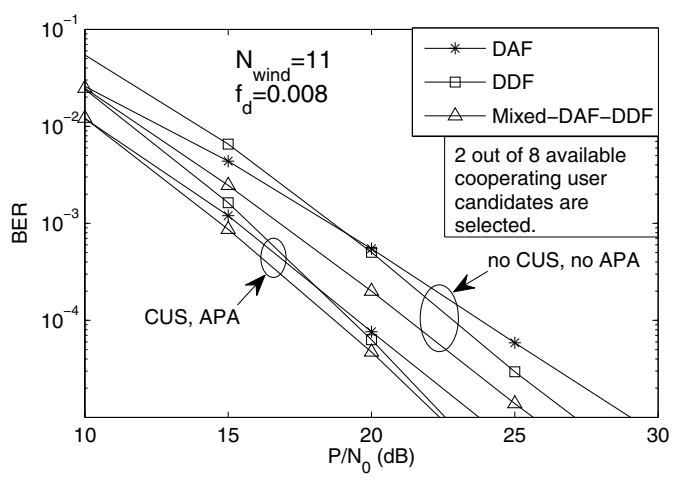

Fig. 7. Performance improvement by the joint CPS and CUS for the DQPSK modulated user-cooperative cellular uplink employing the MSDSD in a Rayleigh fading channel associated with $f_{d}=0.008$ and $v=3$, where 2 out of 8 cooperating user candidates are activated.

The BER performance of our proposed hybrid cooperative cellular uplink, where $M_{r}=2$ out of $\mathcal{P}_{\text {cand }}=8$ cooperating MSs are activated, is portrayed in comparison to that of its DAF- and DDFaided counterparts in Fig. 7. Remarkably, as demonstrated by Fig. 7, the hybrid cooperative system outperforms both the DAF- and DDFaided systems, regardless of whether the joint-CPS-CUS-APA scheme is employed. These conclusions remain valid across a wide SNR range of our interest, although the performance advantage of the hybrid scheme over the latter two systems decreases in the context of the joint-CPS-CUS-APA scheme. Furthermore, as the SNR increases, the DDF-aided system is expected to become superior to the other two systems, since the DDF-assisted system performs best, when error-free transmissions can be assumed between the source MS and the RS. By contrast, if the SNR is low, the DAF-aided system performs best amongst the three. More specifically, given a BER target of $10^{-5}$, the resource-optimized hybrid cooperative system is capable of achieving a performance gain as large as $6.5 \mathrm{~dB}$ over its conventional selective DDF-aided counterpart dispensing with any cooperative resource optimization, which is reduced to $5 \mathrm{~dB}$ when compared to the non-optimized DAF-aided system. In addition to the performance advantage of the joint-CPS-CUS-APA-aided hybrid cooperative system, the overall system complexity becomes moderate in comparison to that of DDF-aided system, since only half of the activated MSs have to decode and re-encode the received signal prior to forwarding it. Therefore, the proposed hybrid cooperative system employing the joint-CPS-CUS-APA scheme is capable of achieving an attractive performance, despite maintaining a moderate overall system complexity.

\section{CONCLUSION}

In this paper, the theoretical performance analysis of both the DAF- and selective DDF-aided cooperative systems was carried out, based on which we investigated the resource allocation of the DAF- and selective DDF-aided cooperative cellular uplinks in a comparative way. For the sake of enhancing the achievable end-to-end performance, a resource-optimized hybrid cooperative system was proposed by exploring the complementarity of the DAF and DDF schemes, where a number of cooperating MSs may be adaptively selected from the available MS candidate pool. Furthermore, the cooperative protocol employed by a specific cooperating MS may also be adaptively selected in the interest of achieving the best possible BER performance.

\section{REFERENCES}

[1] G. J. Foschini and M. J. Gans, "On limits of wireless communications in a fading environment when using multiple antennas," Wireless Personal Communications, vol. 6, pp. 311-335, Mar. 1998.

[2] L. Hanzo, O. Alamri, M. El-Hajjar, and N. Wu, Near-Capacity MultiFunctional MIMO Systems: Sphere-Packing, Iterative Detection and Cooperation. Wiley, 2009.

[3] J. N. Laneman, D. N. C. Tse, and G. W. Wornell, "Cooperative diversity in wireless networks: Efficient protocols and outage behavior," IEEE Transaction on Information Theory, vol. 50, pp. 3062-3080, Dec. 2004.

[4] T. Himsoon, W. Su, and K. J. R. Liu, "Differential transmission for amplify-and-forward cooperative communications," IEEE Signal Processing Letters, vol. 12, pp. 597-600, Sept. 2005.

[5] T. S. Rappaport, Wireless Communications Principles and Practise. Pearson Education Asia Limited and Publishing House of Electronics Industry, second ed., 2002.

[6] L. Lampe, R. Schober, V. Pauli, and C. Windpassinger, "Multiple-symbol differential sphere decoding," IEEE Transactions on Communications, vol. 12, pp. 1981-1985, Dec. 2005.

[7] L. Wang and L. Hanzo, "Multiple-symbol differential sphere detection for amplify-and-forward cooperative transmission," submitted to IEEE Vehicular Technology Conference, Fall, 2009 (VTC'09 Fall).

[8] M. K. Simon and M. S. Alouini, "A unified approach to the probability of error for noncoherent and differentially coherent modulations over generalized fading channels," IEEE Transactions on Communications, vol. 46, pp. 1625-1638, Dec. 1998.

[9] Q. T. Zhang and X. W. Cui, "A closed-form expression for the symbolerror rate of M-ary DPSK in fast rayleigh fading," vol. 53, pp. 1085 1087, 2005.

[10] J. G. Proakis, Digital Communications. 4th edition, New York, NY: Mc-Graw-Hill, 2000.

[11] T. Himsoon, W. P. Siriwongpairat, W. Su, and K. J. R. Liu, "Differential modulations for multinode cooperative communications," IEEE Transactions on Signal Processing, vol. 56, pp. 2941-2956, July 2008. 